\title{
Mohamed SFIA
}

Département de sociologie, Université de Montréal

(1988)

\section{"Réflexions autour de la situation dans le monde arabo-musulman”}

\author{
Un document produit en version numérique par Jean-Marie Tremblay, bénévole, \\ professeur de sociologie au Cégep de Chicoutimi \\ Courriel: jean-marie tremblay@uqac.ca \\ Site web pédagogique : http://www.uqac.ca/jmt-sociologue/ \\ Dans le cadre de: "Les classiques des sciences sociales" \\ Une bibliothèque numérique fondée et dirigée par Jean-Marie Tremblay, \\ professeur de sociologie au Cégep de Chicoutimi \\ Site web: http://classiques.uqac.ca/ \\ Une collection développée en collaboration avec la Bibliothèque \\ Paul-Émile-Boulet de l'Université du Québec à Chicoutimi \\ Site web: http://bibliotheque.uqac.ca/
}


Cette édition électronique a été réalisée par Jean-Marie Tremblay, bénévole, professeur de sociologie au Cégep de Chicoutimi à partir de :

Mohamed SFIA

“Réflexions autour de la situation dans le monde arabo-musulman.”

Un article publié dans la revue Cahiers de recherche sociologique, vol. 6, no 1, printemps 1988, pp. 115-130. Montréal: Département de sociologie, UQÀM. Numéro intitulé: "L'économie mondiale en mutation”.

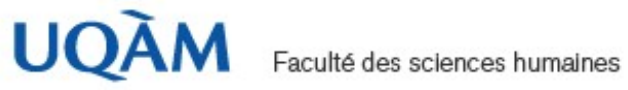

\section{$\rightarrow$ Cahiers de recherche sociologique}

UQAM, Cachier de recherche sociologique

[M. Marcelo Otero, directeur de la revue Cahiers de recherche sociologique, département de sociologie, UQÀM, nous a accordée le 16 mars 2007 sa permission de diffuser le texte intégral de cet article dans Les Classiques des sciences sociales.]

Courriel : otero.marcelo@uqam.ca

Polices de caractères utilisée :

Pour le texte: Times New Roman, 14 points.

Pour les citations : Times New Roman, 12 points.

Pour les notes de bas de page : Times New Roman, 12 points.

Édition électronique réalisée avec le traitement de textes Microsoft Word 2004 pour Macintosh.

Mise en page sur papier format : LETTRE (US letter), 8.5'’ x 11'’)

Édition numérique réalisée le 28 mars 2007 à Chicoutimi, Ville de Saguenay, province de Québec, Canada. 


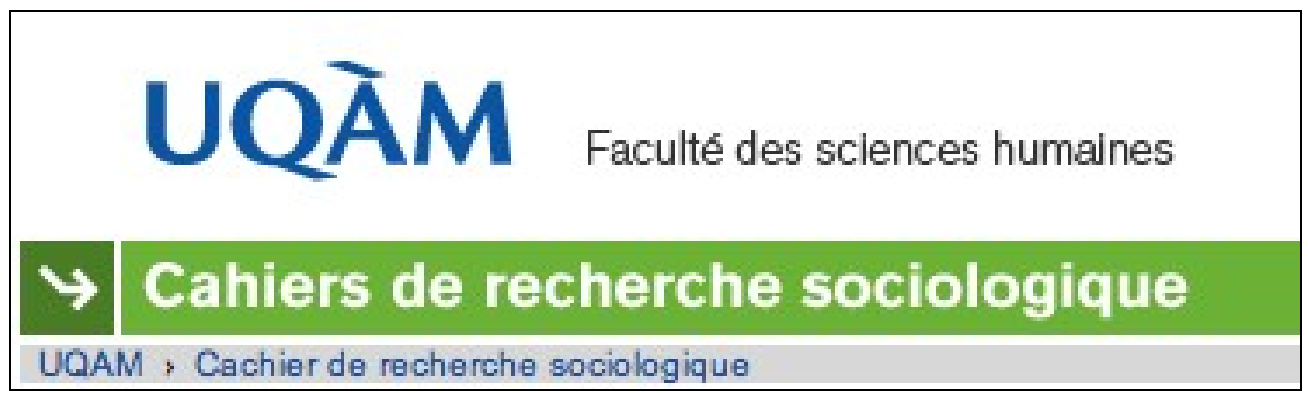

[M. Marcelo Otero, directeur de la revue Cahiers de recherche sociologique, département de sociologie, UQÀM, nous a accordée le 16 mars 2007 sa permission de diffuser le texte intégral de cet article dans Les Classiques des sciences sociales.]

Courriel : otero.marcelo@uqam.ca 


\section{Mohamed SFIA (1988)}

\section{"Réflexions autour de la situation dans le monde arabo-musulman"}

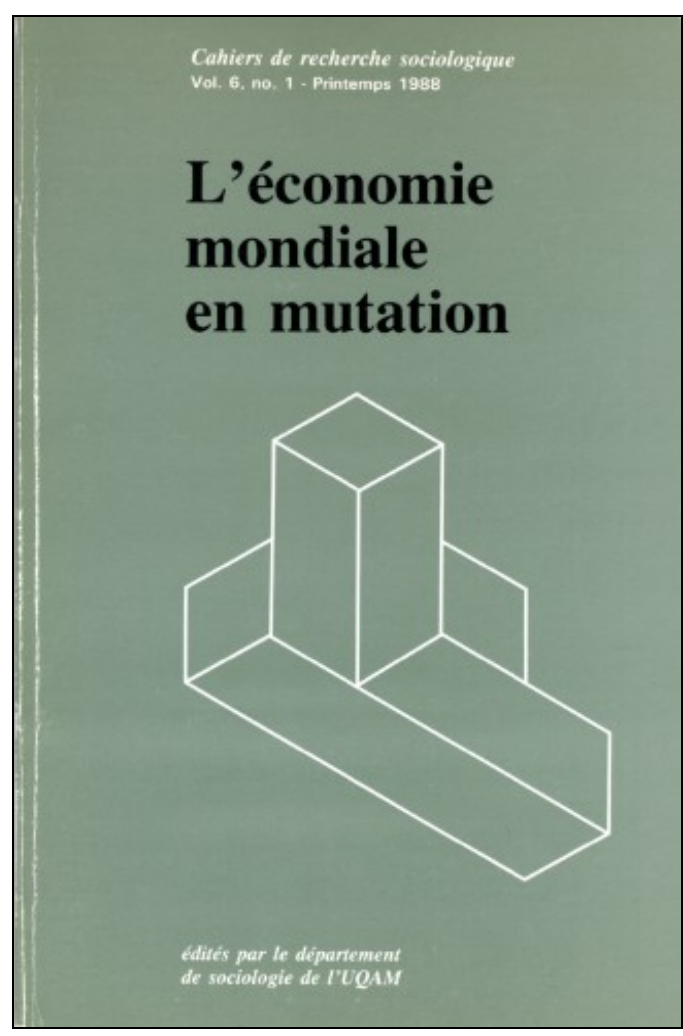

Un article publié dans la revue Cahiers de recherche sociologique, vol. 6, no 1, printemps 1988, pp. 115-130. Montréal: Département de sociologie, UQÀM. Numéro intitulé: “L'économie mondiale en mutation”. 


\section{Table des matières}

\section{Introduction}

1. De l'unité d'analyse

Qu'est-ce qu'une entité "significative" ? Mais quel "domaine islamique"?

2. Idéologie islamiste et perspective fondamentaliste

3. Islamisme et lutte contre la domination 
Mohamed SFIA (1988)

"Réflexions autour de la situation dans le monde arabo-musulman”

Un article publié dans la revue Cahiers de recherche sociologique, vol. 6, no 1, printemps 1988, pp. 115-130. Montréal: Département de sociologie, UQÀM. Numéro intitulé: "L'économie mondiale en mutation".

\section{Introduction}

$\underline{\text { Retour à la table des matières }}$

"Réflexions autour" : c'est en effet ce qu'on trouvera dans les pages qui suivent ; il ne s'agit donc pas d'un "balayage" systématique et détaillé du "champ" qui nous intéresse ici, c'est-à-dire du paysage (économique, politique, culturel, etc.) qui s'offre au regard de celui qui entreprend d'observer raire arabo-musulmane.

\section{De l'unité d'analyse}

L'on doit tout \&abord évoquer le problème bien connu que pose, à quiconque se penche sur une partie donnée du monde, l'opération de délimitation de celle-ci : quel espace est-il légitime de constituer en objet d'observation ? Interrogation qui, d'ailleurs, renvoie à la question générale du découpage : lorsque l'on veut étudier le monde par grandes régions, comment le découper en parties significatives?

\section{Qu'est-ce qu'une entité "significative" ?}

Défendant le paradigme dit "du système mondial", Immanuel Wallerstein et ses collaborateurs écrivent: "The premise is that the arena within which social action takes place and social change occurs is not 
"society" in the abstract, but a definite 'world' a spatio-temporal whole..." 1 . On voit quelle est la démarche des auteurs : se demandant quelle "unité d'analyse" il convient d'identifier, ils adoptent le point de vue selon lequel un espace donné doit, pour pouvoir être légitimement traité comme étant une unité d'analyse, avoir la propriété d'être le lieu d'une action sociale et d'un changement social donnés.

Il y a là un procédé de repérage qui possède certaines vertus heuristiques. On peut, en particulier, le transférer du domaine où Wallerstein et son équipe l'ont mis en oeuvre, celui de l'analyse du système mondial, au domaine de l'observation des grands secteurs du globe. On dira alors que, pour avoir méthodologiquement le droit de découper dans l'étendue planétaire un ensemble régional donné, il faut pouvoir dire de cet ensemble qu'il constitue le cadre d'une action sociale (ou historique) et d'un changement social (ou historique) spécifiques ${ }^{2}$.

Parler ainsi \&action sociale-historique et de changement socialhistorique, c'est voir dans la scène où se déploient cette action et ce changement un complexe de pôles, de secousses, de flux et d'ondes de choc: en un mot, c'est y voir un champ de forces; quelque chose comme un "milieu", une "matière", parcourus de courants, de processus, de mouvements et d'interactions; le tout tournant autour d'un ou de plusieurs centres de gravité.

Si c'est ce genre de dynamique qui à un certain moment constitue une aire donnée en champ de forces, il s'ensuit que l'évolution de la dynamique en question, évolution dont la nécessité est inscrite dans l'ordre des choses, modifie au fil du temps, dans certaines limites, les contours, l'étendue, bref la consistance de l'aire considérée.

1 T. K. Hopkins et Immanuel Wallerstein, "Patterns of Development of the Modem World-System", Review, vol. 1, no 2, automne 1977, pp. 111-145, en particulier p. 112. (Les autres collaborateurs qui ont pris part à la préparation de ce travail sont N. Bousquet, N. Dyson-Hudson, P. McMichael et D. Tomich.)

2 "Ce qui est visé ici", dit Castoriadis, "est l'élucidation de la question de la société et de la question de l'histoire, questions qui ne peuvent être entendues que comme une seule et la même : la question du social-historique "(L'institution imaginaire de la société. Paris, Seuil, 5e édition, 1975, p. 233). 
Pour ce qui est de l'espace qui nous occupe ici, il importe de distinguer trois phases dans la période qui s'est écoulée depuis la Deuxième Guerre mondiale :

1) De la formation de la Ligue des États arabes en $1945{ }^{3}$ à la nationalisation du canai de Suez par Nasser en 1956, c'est le Moyen-Orient qui est le champ de forces.

2) De 1956 à la mort de Nasser en 1970, ce qui s'institue en champ de forces, c'est le Monde arabe.

3) De 1970 à nos jours, c'est le domaine islamique - a-t-on tendance à dire - qui prend la relève.

\section{Mais quel "domaine islamique" ?}

\section{$\underline{\text { Retour à la table des matières }}$}

"Semblable à une écharpe qui entourerait le globe au niveau de l'Afrique et de l'Asie, l'aire islamique s'étire de l'Atlantique au Pacifique, du Maroc à l'Indonésie" 4. Ceinture immense, peuplée d'un milliard d'êtres humains environ (selon certaines estimations), et qui, malgré certains facteurs d'unité, est par trop hétérogène à bien des égards. Ce qui, au sein de cet espace, présente aujourd'hui les caractéristiques d'une caisse de résonance au sens le plus fort ; ce qui y apparaît comme le cadre dans lequel les ondes de choc atteignent leur intensité maximale; ce qui, en somme, fonctionne véritablement comme un champ de forces, c'est l'ensemble arabo-iranien ; lequel peut par conséquent être tenu pour le "noyau dur" du monde musulman. C'est de ce noyau que nous parlons ici lorsque nous disons

3 Les États fondateurs étaient l'Égypte, la Syrie, l'Irak. le Liban. la Transjordanie, l'Arabie Saoudite et le Yémen. Les autres États arabes adhéreront à la Ligue plus tard, au rythme des indépendances.

4 P. Balta, "Le monde islamique", dans E. Camarin (dir.), L'État du TiersMonde, Paris, La Découverte et le Comité Français Contre la Faim ; Montréal : Boréal Express, 1987, 337 p., p. 24. 
"monde arabo-musulman" : il s'agit, si l'on veut, du monde arabomusulman au sens étroit (dans l'un des sens étroits possibles).

\section{Idéologie islamiste et perspective fondamentaliste}

$\underline{\text { Retour à la table des matières }}$

Que se passe-t-il de Particulièrement important dans cette zone ?

Ce qui attire l'attention en ce moment, c'est évidemment cette fameuse "montée de l'islam". Une littérature abondante, et qui ne cesse de s'accroître à un rythme extrêmement rapide, évoque de mille et une façons le "retour de l'islam" 5.

D'entrée de jeu, des mises au point s'imposent, relativement à la terminologie, et, partant, aux objets désignés.

En premier lieu, l'islam étant une religion, parler dune "poussée", d'un "renouveau", etc., de l'islam prête à confusion. En effet, ce à quoi on a affaire, ce n'est pas seulement la reviviscence d'une ferveur religieuse, ce n'est pas seulement l'intensification de la foi et le développement de la pratique. Ce à quoi on assiste, c'est bien plus que cela, ou, du moins, c'est autre chose : c'est la mobilisation de l'islam comme idéologie, la mise à contribution d'une religion à des fins d'action sur le monde. Cette idéologie-là, et plus largement le mouvement social qui la porte, c'est l'islamisme ${ }^{6}$.

5 C'est le titre de l'ouvrage de B. Lewis, Le retour de l'islam. Paris, Gallimard, trad. de l'angl., 1985, 424 p.

6 Parmi ceux qui ont adopté la terminologie appropriée figurent, entre autres, les coauteurs du numéro spécial de la revue SOU'AL (Paris) publié en avril 1985 (no 5) sous le titre L'islamisme aujourd'hui, ainsi que B. Étienne, L'islamisme radical, Paris, Hachette, 1987, 366 p. 
En second lieu, deux vocables sont couramment employés, souvent indifféremment, à propos de ce mouvement : intégrisme et fondamentalisme. En fait, ces termes désignent des phénomènes différents.

L'Intégrisme peut être défini de la manière suivante :

"À l'intérieur d'un mouvement religieux, attitude de fixation et de durcissement opposée à tout développement et à toute évolution (L'intégrisme catholique, qui fut particulièrement combatif sous Pie X [1903-19141 au temps du modernisme, a connu une nouvelle flambée après Vatican II [1962-1965]) ${ }^{7}$.

On le voit : l'intégrisme est, au sens propre, une réaction conservatrice réaction à une tentative d'aggiornamento, c'est-à-dire d'adaptation de la religion à l'évolution ; réaction destinée à faire du dogme un instrument de sauvegarde du statu quo ou de régression vers le passé, dans le but d"'arrêter le temps" ou de "rebrousser chemin". L'intégrisme est donc définissable comme étant l'attitude qui consiste à refuser le changement, à placer ce refus sous le signe d'un retour intégral à la religion, à procéder à une lecture pointilliste et formaliste du corpus doctrinal de base, à dégager des préceptes "purs et durs" et à en prôner la stricte application.

Le fondamentalisme, par contre, s'il implique lui aussi un te retour", relève d'une logique fort différente. Ici, pas de refus de l'histoire, pas de rejet du présent. Il y a au contraire acceptation de la nécessité de faire face aux problèmes du hic et nunc. Le fondamentaliste est même, au départ, quelqu'un qui opère une sorte de franche plongée dans les questions de l'heure, les prend à bras-le-corps, en est submergé. Et sa façon d'aborder ces questions consiste à chercher à inventer, à innover, à réformer. Le fondamentaliste est un réformiste; donc, à la limite, tout le contraire d'un intégriste. Mais il refuse de se laisser entraîner à adopter les solutions toutes faites que suggère, qu'impose, l'ordre établi : ces solutions, véritable "prêt-à-porter", lui paraissent, pour telle ou telle raison, inadéquates, fausses, inacceptables. Il entreprend alors une quête des fondements, des valeurs fondamentales,

7 Article "Intégrisme" dans le Grand Dictionnaire Encyclopédique Larousse (GDEL) en dix volumes, Paris, 1984. 
susceptibles de permettre la redéfinition du point de départ, de la base sur laquelle l'on peut édifier, "organiser la vie", "changer la vie". Généralement - quoique pas nécessairement -, ce genre de démarche mène à un "retour aux sources", notamment aux sources religieuses.

Bien entendu, en tentant de cerner, comme nous venons de le faire, la nature de l'intégrisme et celle du fondamentalisme, nous avons, comme toujours en pareil cas, construit des types idéaux. Dans la réalité "impure", ce qu'on rencontre ce sont des combinaisons, le plus fréquemment. D'où toute une gamme de tendances : à un extrême, des mouvements essentiellement intégristes ; à l'autre extrême, des mouvements massivement fondamentalistes; et entre les deux, des te composés" alliant, à des doses variables, de l'intégrisme et du fondamentalisme.

Pour des raisons sans doute historiques, l'intégrisme tend à être mieux identifié que le fondamentalisme, le résultat étant la propension à assimiler paresseusement tout "retour aux racines" à de l'intégrisme. Pourtant, pour l'observateur du panorama des idéologies dans le monde, il y a lieu de distinguer. Car le fondamentalisme est un phénomène spécifique, qui est bien présent dans l'arène des luttes, et qu'il est nécessaire d'appréhender dans ce qu'il a de caractéristique et de significatif. Ainsi, il n'est pas inutile de rappeler que la pensée de Mao Tsé Toung, et avec elle l'ensemble du projet de la Révolution chinoise, ont parfois été analysés comme ressortissant au fondamentalisme. La théologie de la libération est aussi, de son côté, l'un des fondamentalismes importants de notre époque. Fondamentaliste est également le courant formé par Ivan Illich et par ceux qui creusent le même filon que lui, puisque l'inspiration principale de ce courant réside dans la critique qu'il fait de l'hétéronomie au nom du principe d'autonomie considéré comme valeur fondamentale. Etc. ${ }^{8}$.

L'islamisme d'aujourd'hui est, dans son ensemble, fondamentaliste, et non pas intégriste ${ }^{9}$. Car, pour l'essentiel, cet islamisme n'est pas

8 On voit qu'il existe des fondamentalismes laïques : le maoïsme, l'orientation illichienne (nonobstant le fait qu'Illich ait été un prêtre antérieurement).

9 "Il faut rappeler", note B. Étienne, "que le moi 'intégrisme' a été inventé au début du XXe siècle pour qualifier un certain type de catholicisme ; appliquer 
une réaction, obsédée par une exigence de "fixation-durcissement" des dogmes religieux menacés par l'évolution, mais une réponse aux défis du temps présent, et une réponse à tonalité réformiste ${ }^{10}$. Certes, l'islamisme n'est pas homogène, et bien des visées plus ou moins dissemblables y coexistent : de l'intégrisme se mêle ici et là au fondamentalisme, en proportions changeantes. Mais c'est la logique fondamentaliste qui domine au sein du vaste mouvement islamiste 11.

Il fallait souligner ces précisions, pour faire voir que l'effervescence islamiste dont l'aire arabo-musulmane est actuellement le théâtre n'est pas une espèce d'explosion mystique subite, une sorte de raptus religieux soudain, irrationnel, inintelligible, mais un mouvement social animé d'un projet déterminé d'action transformatrice : transformation d'un système donné de rapports de forces. C'est ce dont il va être question ci-dessous.

ce mot à l'Islam, c'est faire preuve d'européo-centrisme, alors que, au mieux, l'objet n'est pas comparable" (Op. cit., p. 168).

10 Kadhafi est un cas typique de fondamentalisme islamiste. Son point de départ, c'est la recherche de solutions à des problèmes aussi "terrestres" que ceux de la libération nationale, du développement économique, de la justice sociale, de la démocratie politique, etc. Écartant, comme il le dit, la solution libérale et la solution marxiste, il essaie de trouver dans l'islam le plus dépouillé, celui du Coran (et de rien d'autre), les fondements d'une politique.

11 Les problèmes de terminologie, déjà compliqués quand il s'agit de la langue française seulement, le deviennent encore plus lorsque l'on passe à l'anglais. Là, les mots integrism et integralism, quoiqu'ils existent, ne s'emploient guère. Si bien que les auteurs qui veulent faire la distinction entre intégrisme et fondamentalisme se trouvent souvent obligés de recourir au vocable fundamentalism et de lui adjoindre un qualificatif. Ainsi, F. Ajami, appelle conservative fundamentalism ce que nous appelons "intégrisme" et radical fundamenialism ce que nous appelons "fondamentalisme". Voir son ouvrage The Arab Predicament : Arab Political Thought and Practice since 1967, Cambridge, Cambridge University Press, 1981, 220 . 


\section{Islamisme et lutte contre la domination}

$\underline{\text { Retour à la table des matières }}$

Pourquoi donc cette vague islamiste ?

Le danger, face à une telle interrogation, est de s'engager, pour chercher une réponse, dans la voie de l'essentialisme (ou substantialisme). Cela consistera, en l'occurrence, à postuler une essence (ou substance) de l'Islam, une nature immuable du musulman, et à voir dans le mouvement islamiste la manifestation de cet "invariant": quelque chose comme l'"éternel musulman", comme d'autres - ou les mêmes - parlent de l"'éternel féminin", etc. Pour une part considérable, le discours tenu aujourd'hui - tout comme hier, du reste -, ici et là, sur l'Islam, l'islamisme et les musulmans, est un discours largement essentialiste.

À cette approche il faut, résolument, en opposer une autre, toute différente. "L'apport majeur de ce qu'il faut bien appeler la révolution structuraliste", dit Bourdieu, "a consisté à appliquer au monde social un mode de pensée relationnel, qui est celui de la mathématique et de la physique moderne et qui identifie le réel non à des substances mais à des relations" 12 . Mettre en oeuvre, dans l'observation de l'islamisme, ce .. mode de pensée relationnel", tel est le principe de méthode que l'on affirmera ici \&entrée de jeu.

Il apparaît alors qu'il existe effectivement un réseau de rapports qui ont pesé d'un poids déterminant sur le "destin" de l'espace arabomusulman" : ce sont les rapports qu'il entretient avec l'Occident.

12 P. Bourdieu, Choses dites, Paris, Minuit, 1987, p. 150. 
L'entité arabo-musulmane? L'Occident ? Ce sont des culturescivilisations ${ }^{13}$, des ensembles ethno-culturels - si l'on préfère - , qui occupent chacun une position donnée dans la structure du système mondial ou, à d'autres moments de l'histoire, dans la structure du système régional dont ils faisaient partie (structure complexe : économique, politique, culturelle, etc.). Des réticences s'expriment parfois, on le sait, à l'encontre de ce genre de découpage de la réalité. Elles émanent généralement de ceux qui, en vertu surtout dune certaine interprétation du marxisme, ne considèrent comme significatifs que les rapports entre classes, entre modes de production, entre États à la rigueur. C'est qu'ils oublient de cultiver la vigilance nécessaire à l'égard des pièges du réductionnisme : la différenciation sociale est un phénomène multidimensionnel (il y a une différenciation selon l'axe des classes sociales, une différenciation selon l'axe des sexes, une différenciation selon l'axe ethno-culturel, une différenciation selon l'axe de l'âge, etc.), et rien n'autorise à occulter, ou à traiter comme un épiphénomène, telle ou telles forme(s) de différenciation, pour tout réduire à l'une d'entre elles; car l'on est alors guetté par les simplifications de l'économisme, du culturalisme, etc.

La région arabo-musulmane présente, par rapport aux autres parties de ce qu'on nomme de nos jours le "Tiers-Monde", la particularité d'avoir été très tôt mise en contact, et étroitement, avec l'Europe. Ce contact s'est en fait établi immédiatement, c'est-à-dire au moment même de la naissance de la civilisation arabo-islamique, aux Vlle et VIlle siècles. Mais un pas capital a été franchi aux Xle et XIle siècles avec la mise en branle du mouvement des Croisades, lesquelles allaient marquer en profondeur, et durablement, la conscience collective occidentale tout comme la conscience collective arabo-musulmane.

Chez les Européens s'est de bonne heure développée la "peur du sarrazin", qui devait devenir dans l'ensemble de l'Occident la plus grande des deux principales "peurs de l'autre" (la deuxième de ces peurs, bien plus tardive et beaucoup moins obsédante, étant la crainte du "péril jaune"). Le "réflexe de croisé" s'est profondément ancré, et

13 On laissera de côté, ici, le problème de la différence entre culture et civilisation. 
pour longtemps, dans l'univers mental judéo-chrétien ${ }^{14}$, ce qui a fait parler, à ce propos, d'une "idéologie de longue durée" ${ }^{15}$. Et l'ombre de la croisade n'est-elle pas réapparue lorsque l'Ouest a eu affaire à Nasser, tout comme elle se profile maintenant à propos du conflit avec Khomeini, etc. ? 16.

Les Arabo-musulmans, de leur côté, sont littéralement hantés par le souvenir des Croisades. Ici, laissons longuement la parole à Amin Maalouf, auteur de Les Croisades vues par les Arabes :

"On est souvent surpris de découvrir à quel point l'attitude des Arabes, et des musulmans en général, à l'égard de l'Occident reste influencée, aujourd'hui encore, par des événements qui sont censés avoir trouvé leur terme il y a sept siècles..."

Et d'ajouter un plus loin :

"Dans un monde musulman perpétuellement agressé, on ne peut empêcher l'émergence d'un sentiment de persécution, qui prend, chez certains fanatiques, la forme d'une dangereuse obsession : n'a-t-on pas vu, le 13 mars 1981, le Turc Mehemet Ali Agca tirer sur le pape après avoir expliqué dans une lettre : " J'ai décidé de tuer Jean-Paul II, commandant suprême des croisés ». Au-delà de cet acte individuel, il est clair que l'Orient arabe voit toujours en l'Occident un ennemi naturel. Contre lui, tout acte hostile, qu'il soit politique, militaire ou pétrolier, n'est que revanche légitime. Et l'on ne peut douter que la cassure entre ces deux mondes date des croisades, ressenties par les Arabes, aujourd'hui encore, comme un viol" . 17

14 Voir en particulier, l'étude classique de P. Alphandéry et A. Dupront, La Chrétienté et l'idée de croisade, Paris, Albin Michel, 2 volumes, 1954 et 1959, 248 p. et 336 p.

15 P. Rousset, Histoire d'une idéologie : la croisade, Lausanne, l'Âge d'Homme, 1983, p. 213.

16 Voir par exemple les remarques de B. Étienne au début de son livre déjà cité. Il écrit entre autres: "Il n'est pas possible d'aborder l'Islam sans avoir à l'esprit toute la masse accumulée d'informations qui constituent la mémoire d'une société comme la nôtre : les Croisades, la mission civilisatrice de la France au Moyen-Orient depuis François 1er, la guerre d'Algérie, la présence massive d'immigrés, etc., car l'imaginaire occidental s'est construit une hostilité dramatique à l'encontre de l'Islam". (p. 7).

17 A. Maalouf, Les Croisades vues par les Arabes, Paris, J.-C. Lattès, 1983, 303 p., p. 283. 
Puis le monde arabo-musulman a connu une décadence qui n'a fait que s'accentuer de siècle en siècle. Une nouvelle "effraction" l'a secoué à la fin du VIlle siècle : l'expédition de Bonaparte en Égypte en 1798-1801. Celle-ci a fait l'effet, dit-on couramment, d'un "coup de tonnerre" : le monde arabo-musulman a pris conscience de son retard ; pire encore, il a compris qu'il était désormais une proie, que l'Autre, plus développé et plus fort, pouvait convoiter, attaquer, soumettre.

C'est ce qui s'est effectivement produit ensuite : les sociétés arabomusulmanes ont petit à petit subi toutes les formes possibles de colonisation.

Si l'on entreprend de rappeler les grands moments de la résistance qui a été opposée à cette mainmise coloniale, on doit évoquer, en simplifiant bien sûr, les étapes suivantes :

1) À partir du milieu du XIXe siècle a commencé tout un travail de questionnement, de réflexion et de recherche, qui a pris l'allure d'un "examen de conscience" face à la crise provoquée par le "défi européen". Il s'est agi \&un réformisme qui tentait de puiser des éléments à la fois dans le modèle occidental et dans la tradition autochtone, soit religieuse soit séculière. En Iran, l'inspiration islamique l'a massivement emporté. Dans les pays arabes, le mouvement, qui a pris le nom de "Nahda" (Réveil, Renaissance), a - tout en mettant aussi l'accent sur l'impératif de la fidélité à la tradition et notamment à la religion laissé une plus grande place que l'Iran à ridée de la nécessité de se mettre à l"'école de l'Occident".

2) Au cours de la majeure partie de la première moitié du XXe siècle, le monde arabo-musulman a, en quelque sorte, commencé à donner à cette fermentation intellectuelle les suites qu'elle impliquait. C'est à ce moment-là que se sont déroulées, après divers sursauts au XIXe siècle, les premières grandes luttes modernes contre la tutelle européenne, et qu'ont été obtenus les premiers résultats : Révolution constitutionnelle iranienne de 1905-1911; indépendance de l'Égypte en 1922 ; mise en oeuvre d'une politique nationale par Reza Chah Pahlevi en Iran à partir de 1925 ; indépendance de l'Irak en 1930 ; éva- 
cuation militaire de l'Égypte (sauf la zone du canal de Suez) par l'Angleterre en 1936 ... pour ne citer que ces jalons-là.

3) La dernière décennie de la première moitié de ce siècle a été décisive, parce que les Iraniens d'une part et les Arabes d'autre part ont subi deux lourdes défaites. Iran : en 1941, l'Angleterre et l'Union soviétique se sont entendues pour attaquer le pays, déposer et exiler Reza Chah Pahlevi -dirigeant dans lequel le peuple iranien s'était largement reconnu -, et occuper le territoire pour des années (en 1942 des troupes américaines se sont jointes aux forces d'occupation). Pays arabes : en 1948 a eu lieu cet événement considérable qu'a été la création de l'État d'Israël, création qui a été perçue - et est toujours perçue - par les Arabo-musulmans comme une véritable catastrophe, car ils y ont vu un fait colonial qui leur a été imposé en pleine période de décolonisation ; fort parlante est à cet égard l'expression "morsure israélienne" qui a été employée 18.

4) La phase suivante a été celle d'une double réponse nationalitaire ${ }^{19}$. En Iran, ce fut ce nationalitarisme qui a produit et porté Mos-

18 Voir G. Corm, Le Proche-Orient éclaté : de Suez à l'invasion du Liban, 19561982, Paris, La Découverte, nouv. éd. mise à jour 1984, p. 194 suiv.

19 C'est M. Rodinson, et surtout A. Abdel-Malek, qui, à la suite d'autres auteurs moins récents, ont eu le mérite d'avoir attiré l'attention sur la différence qu'il y a entre nationalisme et nationalitarisme. Voir A. Abdel-Malek, La dialectique sociale, Paris, Seuil, 1972, 480 p., p. 61 et suiv. De quoi s'agit-il ? Au départ, on est en présence d'un fait simple : le phénomène "nation" produit une idéologie spécifique, quelque chose qu'on peut appeler l"'auto-affirmation nationale". Mais il y a auto-affirmation nationale et auto-affirmation nationale : celle de la nation qui revendique le privilège de la supériorité (d'une forme quelconque de supériorité) et donc un droit de préséance par rapport à d'autres nations, et celle de la nation qui se trouve contrainte d'opposer une résistance au nom du droit minimal de ne pas être dominée. D'une part, l'autoaffirmation nationale de l'Angleterre du white mans burden, de la France de la "mission civilisatrice de la France", des Etats-Unis de la Manifest Destiny, de l'Allemagne du Deutschland über alles, etc.; d'autre part, l'auto-affirmation nationale de l'Inde face à l'Angleterre, de l'Algérie face à la France, du Nicaragua face aux Etats-Unis, de la Tchécoslovaquie face à l'Allemagne, etc. Le moins qu'on puisse dire est que l'on n'a tout simplement pas affaire à la même 
sadegh, lequel a nationalisé en 1951 le pétrole iranien - c'est-à-dire l'Anglo-Iranian Oil Company (AIOC) -, prenant ainsi une initiative fondatrice dans cette région, car personne jusque-là n'y avait osé toucher à un intérêt aussi puissant ; mais en 1953 la CIA organisait contre Mossadegh le coup d'État du général Zahedi et rétablissait au pouvoir le Chah Mohammad Reza, celui-là même que devait abattre la Révolution iranienne de 1979. Dans le domaine arabe, ce fut la prise du pouvoir par les Officiers Libres en Égypte en 1952, la nationalisation du canal de Suez par Nasser en 1956, l'échec de l'attaque anglofranco-israélienne contre l'Égypte la même année, puis le développement de cette lame de fond du nationalitarisme arabe qu'a été le nassérisme ; mais l'agression israélienne de 1967 a brisé cet élan.

5) Le sens qu'ont eu, aux yeux de ce qu'on pourrait appeler le "monde arabo-musulman profond", tous ces événements (ceux de 1951-1953 en Iran, ainsi que leurs suites ; ceux de 1952-1970 dans l'espace arabe, ainsi que leurs suites), ne fait pas de doute : ils ont paru administrer la preuve, encore une fois, qu'un combat très dur se poursuivait, combat dans lequel chaque fois que tel ou tel secteur du mouvement de libération nationale dans l'ensemble arabo-musulman enregistrait une avancée, l'Occident réagissait avec violence pour rétablir son contrôle. "Faisons-nous la moindre tentative pour relever la tête ?, ils nous la ré-enfoncent dans l'eau !" L'Occident avait, depuis des siècles, planté petit à petit ses griffes dans le corps meurtri des

réalité, et que l'on ne peut pas recourir au même terme, nationalisme, pour désigner deux objets aussi différents. On appellera donc "nationalisme" l'idéologie de la nation dominante, et nationalitarisme" l'idéologie de la nation dominée. Mais si l'usage n'a pas consacré une telle distinction, c'est que, dans un rapport de domination, le brouillage des notions n'est pas un phénomène contingent mais un produit nécessaire de ce rapport, puisque le dominant, qui a le pouvoir - matériel et symbolique - d'imposer sa façon de nommer les choses, les nomme de manière à faire méconnaître ce qu'il a intérêt à faire méconnaître. Le dominant fait tout, entre autres, pour créer le maximum de confusion concernant la répartition des rôles entre le bourreau et la victime ici entre la nation bourreau et la nation victime. Le dominant va même plus loin : opérant un renversement des rôles, il réserve à l'idéologie de la nation dominée l'étiquette "nationalisme" - ce dernier portant le stigmate infâmant du "particularisme" - et s'arroge lui-même - nation dominante - le monopole de l'"universalisme", posture noble s'il en est. 
peuples arabo-musulmans - ce qui signifie nécessairement, dans leur âme aussi - et ne les lâchait plus ; tout sursaut pour lui faire lâcher prise se heurtait à la farouche détermination du bourreau d'écraser la victime plus encore que par le passé, à la fois pour continuer à la dominer et pour lui faire payer cher l'audace qu'elle avait eue de se révolter. A partir de cette conviction que telle était bien la logique de la partie qui se jouait, il s'est enclenché dans la zone arabo-musulmane un processus souterrain de recherche " une arme idéologique plus efficace que la précédente, celle du nationalitarisme laïque et moderniste de Mossadegh et de Nasser : une idéologie pouvant permettre une plus grande mobilisation en vue de batailles plus difficiles. Le fondamentalisme islamiste, courant constamment présent dans le monde arabo-musulman depuis longtemps, est alors tout naturellement apparu comme étant le candidat approprié.

L'islamisme est donc la nouvelle forme de la poussée nationalitaire qui est à l'oeuvre depuis longtemps dans le monde arabo-musulman. Dans la période récente, cette poussée est représentée, incarnée, par les trois symboles majeurs que sont Mossadegh, Nasser et Khomeini ${ }^{20}$. Chacun de ces derniers exprime la spécificité d'un moment particulier de l'évolution d'une lutte : la lutte entre les Arabo-musulmans, qui tentent de défaire le rapport de domination-subordination qui existe entre eux et l'Occident, et celui-ci, qui oeuvre constamment à reproduire ce rapport. Le moment Mossadegh a été celui d'un nationalitarisme modéré et moderniste-occidentaliste (la définition qui était donnée de la voie à suivre empruntait massivement au modèle dominant de la modernisation) ; le moment Nasser a été celui d'un nationalitarisme semi-radical et moderniste-occidentaliste également; le moment Khomeini est celui d'un nationalitarisme radical et identitaire (la définition qui est donnée de la voie àsuivre comporte une quête de l'identité propre et une recherche des moyens de rompre avec le modèle dominant). Il y a ainsi une escalade qu'il importe de relever : de

20 Kadhafi s'inscrit dans la même lignée. Mais il ne pouvait jouer un rôle analogue, la Libye étant loin d'avoir le poids qu'ont l'Égypte et l'Iran. 
Mossadegh à Khomeini en passant par Nasser, il y a une exacerbation du conflit 21.

Ce qu'il est particulièrement important de souligner à cet égard, c'est que dès les années cinquante en Iran (après le coup d'État monté par les États-Unis contre Mossadegh en 1953), et dès la fin des années soixante dans le secteur arabe (après la guerre déclenchée par Israël en 1967), un sentiment d'abord d'humiliation, ensuite de colère, enfin de rage pure et simple a envahi les consciences. Sur ce matériau plus ou moins brut, la fonction de l'islamisme a précisément consisté à accomplir un travail d'élaboration qui a transformé le tout en cette rage sacrée dont parle à très juste titre Robin Wright ${ }^{22}$. Dès lors, cette rage soutiendra une détermination d'aller jusqu'au bout, une de ces déterminations qu'exprime mieux que quoi que ce soit le Fire next time de James Baldwin ${ }^{23}$. D'où cette violence qui, dernier recours, et recours obligé, semble ne pas craindre de tendre vers le paroxysme.

Mais cette violence - est-il besoin de le signaler ? - n'est pas seulement un moyen, un moyen employé pour vaincre ou ne pas être vaincu dans la confrontation. Elle est aussi, bien sûr, un langage. Le message ? "Si la seule manière de nous faire entendre est de ne reculer devant rien, eh ! bien, soit ! Il. Tout comme l"'autre" crie : "Faut-il faire la folle pour être entendue ?" 24.

Radical, l'islamisme l'est de deux façons. D'un côté, il se présente non comme une contestation ponctuelle, une critique sélective adressée à certains aspects du "système", mais comme un refus global. Ce

21 "Identitaire" : il y en aurait long à dire sur cette problématique de l'"'identité", que l'on tend à redécouvrir actuellement. Mais il n'est pas possible de le faire dans le cadre limité de cet article. Contentons-nous donc de renvoyer ici, entre autres, au volume intitulé L'identité, Séminaire dirigé par C. Lévi-Strauss, Paris, PUF, réédition 1983, 348 p.

22 R. Wright, Sacred Rage : the Wrath of Militant Islam, New York, Simon and Schuster, 1986, 330 p.

23 Il s'agit de son roman traduit en français sous le titre de La prochaine fois le feu.

24 F. Ongarelli-Loup, "Faut-il 'faire la folle' pour être entendue ?", Annales $d u$ Centre de Recherche Sociale (Genève), no 24, octobre 1987, 164 p. 
que Michel Foucault faisait remarquer au sujet de la secousse iranienne de 1979 peut s'appliquer à l'ensemble de la tendance islamiste :

"En Iran, le sentiment national a été extrêmement vigoureux : le refus de la soumission à l'étranger, le dégoût devant le pillage des ressources nationales, le rejet d'une politique étrangère dépendante, l'ingérence américaine partout visible ont été déterminants pour que le chah soit perçu comme un agent de l'Occident. Mais le sentiment national n'a été, à mon avis, qu'une des composantes d'un refus plus radical encore : le refus par un peuple, non pas simplement de l'étranger, mais de tout ce qui avait constitué, depuis des années, des siècles, son destin politique" 25 .

De l'autre côté, l'islamisme a fait ce pas considérable, extrêmement difficile à franchir pour tout dominé, qui consiste à rejeter les règles du jeu que le dominant impose. Il y a ainsi toute une symbolique par laquelle le mouvement islamiste fait tout ce qu'il est possible de faire pour être autre que ce que le dominant voudrait qu'il soit. Mais surtout, dans la lutte elle-même, le dominé s'arroge le droit "inouï" de choisir ses moyens. C'est là tout le problème du "terrorisme" 26. À un dominant qui vise à faire reconnaître comme seules légitimes les méthodes de lutte qui lui conviennent, à un dominant qui décrète le plus tranquillement du monde que recourir à l'aviation la plus sophistiquée pour bombarder des villages est "civilisé" tandis tandis que le Il terrorisme" est "sauvage", le dominé rétorque : "La prise d'otages est la bombe atomique du pauvre" 27.

À la revendication de liberté qui émane des profondeurs du monde arabo-musulman, l'Occident réplique sur un mode parfaitement connu : celui du refus de comprendre. Il y a toujours, dans l'attitude de l'oppresseur face à la lutte de l'opprimé, cette réaction de surdité-

25 C. Brière et P. Blanchet, Iran : la révolution au nom de Dieu, suivi d'un entretien avec Michel Foucault, Paris, Seuil, 1979, p. 231.

26 Lequel nécessiterait, à lui seul, de longs développements ; des développements qui devraient repartir, entre autres, des analyses pionnières que Frantz Fanon a proposées du rapport colonisateur-colonisé, dominant-dominé, de la violence dans ce rapport, de la question des moyens, etc. ; analyses qu'on trouve notamment dans Les damnés de la Terre, Paris, Maspero, 1961, 243 p.

27 Un ayatollah iranien, cité par P. Balta, op. cit., pp. 26-27. 
cécité - volontaire ou semi-volontaire - à propos de laquelle on est tenté de parler de quelque chose comme un "syndrome", le syndrome du "Mais que veulent-ils donc ?". "Que veulent-ils donc (ces terroristes arabes, ces fanatiques musulmans) ?" "What does Quebec want ?" "Mais que veulent-elles donc (ces femmes, ces folles) ? ", etc. Expression obstinée de ce refus de comprendre, la question ne fait qu'annoncer la réponse voulue : "Ces gens-là sont incompréhensibles, ils sont irrationnels; ils ne peuvent être mus que par le démon de la folie et du meurtre, ou par un autre qu'eux-mêmes, l'étranger".

C'est qu'il se produit, dans ce genre de situation, une rupture profonde de la communication, ou plus exactement une aggravation de l'impossibilité de communiquer qui est constitutive du rapport de domination-subordination. Il peut être utile d'évoquer, à ce sujet, la distinction que Jean-François Lyotard, réfléchissant à la nature des désaccords, des oppositions, des conflits, propose d'établir entre "litige" et "différend" 28 . À la différence d'un litige, un différend est un conflit dans lequel le point de vue de l'une des parties ne parvient pas à s'exprimer dans l'idiome de l'autre. De sorte que le tort que subit la victime est double : elle est lésée, et en outre elle n'arrive pas à faire reconnaître qu'elle l'est. C'est dans de telles conditions qu'explose parfois la violence : violence ponctuelle de la victime, qui n'est qu'une réaction d'auto-défense, de résistance, face à la violence institutionnalisée du bourreau.

Finalement, ce qui se passe dans le monde arabo-musulman apparaît symptomatique. Symptomatique d'une crise : celle que le monde le monde entier, et pas seulement telle ou telle région - traverse aujourd'hui, et dans laquelle il faut bien voir un dérèglement général, et pas seulement économique. Inégalités, blocages, oppressions, conflits, violences, malentendus : autant d'éléments de cette crise, à laquelle certaines sociétés, certains groupes, cherchent confusément une issue, en se tournant entre autres vers ce refuge qu'est la religion ${ }^{29}$. Pourquoi ? "On cite toujours Marx et l'opium du peuple", répond Foucault.

28 Voir J.-F. Lyotard, Le différend, Paris, Minuit, 1983, 280 p.

29 Que le "retour du religieux" ne soit pas limité à l'aide arabo-musulmane, voilà qui est évident maintenant. Voir à ce propos, par exemple, le numéro spécial 
"La phrase qui précède immédiatement et qu'on ne cite jamais dit que la religion est l'esprit d'un monde sans esprit. Disons donc que l'Islam en cette année 1978, n'a pas été l'opium du peuple, justement parce qu'il a été l'esprit d'un monde sans esprit" 30 .

\section{L'islamisme est un révélateur.}

\section{Mohamed SFIA}

Département de sociologie, Université de Montréal

(89e année, no 928, mars-avril 1987) de la Revue Politique et Parlementaire (Paris) intitulé "Le retour du religieux en politique".

30 M. Foucault, dans C. Brière et P. Blanchet, op. cit., p. 234. 